INDIVIDUAL REPORTS OF DIVIONS/COMMISSIONS \& WORKING GROUPS

\author{
COMMISSIONS OF THE EXECUTIVE COMMITTEE
}

5 Documentation \& astronomical Data

38 Exchange of astronomers

46 Teaching of astronomy

50 Protection of existing \& potential observatory sites 


\section{DOCUMENTATION AND ASTRONOMICAL DATA (DOCUMENTATION ET DONNEES ASTRONOMIQUES)}

PRESIDENT: B. Hauck

VICE-PRESIDENT: O. Dluzhnevskaya

ORGANIZING COMMITTEE: H.A. Abt, M. Bessell, M. Crézé, A.G. Hearn, H. Jenkner, Li Qi-Bin, A. Piskunov, E. Raimond, G. Riegler, W.H. Warren, D. Wells, R. Wielen, G. Wilkins

\section{INTRODUCTION}

The restructuring of our commission, adopted at the last General Assembly, was undoubtedly beneficial and various activities were developed under the aegis of the commission.

Two very important changes have marked the last triennium: the World Wide Web and electronic publishing. The American Astronomical Society is the first to have introduced an electronic version of an astronomical journal and will be followed soon by the Astronomy and Astrophysics Journal. Last February, an ICSU/UNESCO Conference was held in Paris on this topic. A.G. Hearn was the IAU representative. An extensive report of this meeting can also be found on the Web (http://astro.fys.ruu.nl:8000/iau) and the proceedings are available also on the Web (http://www.lmcp.jussieu.fr/icsu/Information/index.html. A Joint Discussion on this very hot topic will be held during the Kyoto General Assembly.

A. Heck and E. Raimond were respectively the IAU representatives to the 19th and 20th International CODATA Conferences (Chambéry, France, 1994; Tsukuba, Japan, 1996).

Many books and papers concerning Commission 5's topics have been published, among them: The IAU Thesaurus, R. Shobbrook (1994); International bibliography of astronomy serials, Huang Bikun (1995); Second reference dictionary of nomenclature of celestial objects, Lortet et al. [61.002.196]; Intelligent information retrieval: the case of astronomy and related space sciences [62.003.021]; Information and On-line Data in Astronomy (D. Egret \& M.A. Albrecht (Eds., 1995)); Network resources for astronomers [62.002.068]. H.A. Abt has published various very interesting papers reflecting his long experience as Editor of the Astrophysical Journal [57.002.92], [58.002.012], [58.002.144], [61.002.057], [62.002.066], [63.002.149], [APJ 455, 407], [PASP 108, 375]. The Star*s family products (Heck et al.) is accessible via http://cdsweb.u-strasbg.fr.

\section{WORKSHOPS, COLLOQUIUMS, CONFERENCES}

Various meetings relating to the scope of Commission 5 were held, of which the following may be mentioned:

Library and Information Services in Astronomy II, Garching 1995 [63.012.055]

Astronomy from Wide-field Imaging, IAU Symposium 161, Potsdam, 1993 [61.012.055]

Astronomical Data Analysis Software and Systems IV, 1994 [R.A. Shaw et al. (Eds.)]

Astronomical Data Analysis Software and Systems V, 1995 [G.H. Jacoby et al. (Eds.)]

Weaving the Web, Strasbourg, 1995 (A. Heck \& D. Egret (Eds.)), Vistas in Astron. 39, 1995.

New Horizons from Multi-Wavelength Sky Surveys, IAU Symposium 179, Baltimore, 1996

International Co-operation in the Dissemination of Astronomical Data, Pulkovo, 1996

[A.G. Hearn and F. Ochsenbein (Eds.); http://www.inasan.rssi.ru/refs/]

\section{REPORTS FROM DATA CENTRES AND NETWORKS}

\subsection{NASA ADC (N.G Roman)}

In February 1996 there were nearly 9000 accesses to our archive. It is clear that the move to an online archive has increased the use of our archive in a major way. This has been the primary change in the 
U.S. Astronomical Data Center but we have also produced a second CD-ROM that contains approximately 60 large, popular catalogues. These include the three Durchmusterungs, the PPM, the Tycho Input Catalogue and the 6C Surveys of Radio Sources. We are preparing standard documents following the CDS format for all new catalogues. In addition, we are preparing Brief Descriptions for all of the older catalogues which do not have them, and standard electronic documents for older catalogues which now have only hard copy or very minimal documentation, and are adding ADC keywords to all journal files which do not already have them. It will take some time before these efforts are completed.

The new WWW home page can be accessed through URL http://adc.gsfc.nasa.gov/ or ftp://adc.gsfc.nasa.gov/pub/adc. From this page, Web users can easily explore the ADC's new FTP site or query a database of descriptions of the catalogues and journal tables.

\subsection{CDS, Strasbourg (F. Genova)}

The development of SIMBAD, the reference database for the identification and bibliography of astronomical objects, was continued. In May 1996, SIMBAD contained 1,225,000 objects (up by $65 \%$ compared to 1990), with more than 3,773,000 object names, 92,000 references from 90 journals $(+60$ $\%$ ) and 1,795,000 object citations. The Paris, Bordeaux and Strasbourg observatories participate in the bibliography scanning, under the responsibility of the Institut d'Astrophysique de Paris. A large effort was devoted to the inclusion of stellar catalogues (PPM, HIC, CCDM) in the context of the HIPPARCOS mission, of the IUE log and to the "cleaning" of the Einstein and older X-ray catalogues, to improve the multiwavelength coverage. A new XWindow menu interface was distributed by the end of 1994, and a WWW interface is foreseen in the near future.

The Catalogue Server displays catalogues and published tables (1550 catalogues and tables). The collection of catalogues is a co-operative action with the other Data Centers. A general standard description of the Tables was established by CDS in 1993; it is now shared by the Data Centers, and the Journal publishers for the electronic publication of Tables. VizieR, developed jointly by the CDS and ESA-ESRIN as a follow-up of the ESIS Catalogue Browser, allows one to query any table or catalogue by any of its fields. The CDS builds the electronic tables for Astronomy and Astrophysics, as part of the publication process, and installs the tables from the AAS CD-ROMs on-line, by agreement with the journal editors.

The CDS is also developing the ALADIN project, an interactive sky atlas, which provides deep images from the digitised sky, with astrometric and photometric calibration, on which information from SIMB AD, from the CDS catalogues and Tables, and from user-provided tables, can be overlaid. It will thus be a major tool for multi-wavelength cross-identification.

The CDS WWW server, (http://cdsweb.u-strasbg.fr/CDS.html) gives access to the Catalogue Server and VizieR, to bibliography information, to documentation, and to some services hosted by the CDS, "Yellow Page" services, such as the Star*s Family, developed by A. Heck, and Astroweb, the TOPBase atomic physics database from the OPACITY project... The CDS also hosts the archives of unpublished data on variable stars (IAU Commission 27). The "Bulletin d'information du CDS" is published twice a year. A hot-line at email "question@simbad.u-strasbg.fr" is organized.

\subsection{Astronomical Data Analysis Center, National Astronomical Observatory of Japan (K. Nakajima)}

1. Development of an observational data archiving system "MOKA".

An archiving system for the ground based observational data obtained at the Okayama Astrophysical Observatory and Kiso Observatory was developed. It is called "MOKA", Mitaka Okayama Kiso Archival system. [62.002.165], [Takata, et al., 1995, Publ. Natl. Astron. Obs. Japan, 4, 9.]

2. Construction of an astronomical catalogue service system: An astronomical catalogue service system which includes WWW and anonymous ftp servers was developed in June, 1995, and is now in operation. A copy of publicly opened data at CDS, Strasbourg, is maintained at Tokyo by mirroring them regularly. [Nakajima et al. 1995, Rep. Natl. Astron. Obs. Japan, 2, 697]. 
3. Development of public dissemination system for astronomical information "PAONET" (Public Astronomical Observatory NETwork): Software system to support transport and display of latest astronomical images at public observatories or science museums [63.014.090].

\subsection{Centre of Astronomical Data, Institute of Astronomy of the Russian Academy of Sciences (INASAN) (O. Dluzhnevskaya)}

The archive now stores an almost complete copy (about 700 entries, excluding big surveys on CDROMs like DSS, Einstein Observatory data, etc.), which is regularly updated. Several well-known astronomical archives and databases stored at INASAN (e.g., ULDA, BDA) are also available. The typical CAD data exchange rate via Internet is $50-100 \mathrm{Mb}$ per day.

The protocol of the project INTAS "Integration of the FSU observatories into international astronomical data network" by CDS as the project coordinator, with CAD and 7 astronomical institutions of the FSU, was signed in the beginning of 1995. The Trieste Astronomical Observatory is also involved in the project. A node of astronomical network has just been established in the CAD, and access of the FSU observatories to international networks (including WWW facilities) is maintained.

CAD prepares new catalogues, archives and databases as well as published tabular data from the main Russian astronomical journals.

Since 1994 CAD has participated in the Spectrum-UV project. Up to now main efforts were concentrated on preparation of the Master Catalogue. This work is now in progress in collaboration with Sternberg Astronomical Institute (Moscow, Russia), the NED group (California, USA), and OAT (Trieste, Italy).

The Catalogue of Star Forming Regions of the Galaxy has been maintained in CAD for several years. The catalogue contains data on structure and radiation at various wavelengths (radio, millimeter, IR etc.) of different objects, associated with high luminosity stars and neighbouring media. The second version of the catalogue is now in preparation. Several new catalogues, e.g., Stellar Mass Catalogue (SMAC), Bibliographical Catalogue of Stellar Polarization (BCSP), Low mass binaries database (LOMBI) and compressed version of the Guide Star Catalog have been recently prepared. We also plan to issue the CD-ROM containing the main catalogues, prepared in FSU countries.

A software for retrieving and visualization of data from the Guide Star Catalogue is implemented and identification of GSC objects with extragalactic objects and Solar System bodies in collaboration with NED/Caltech and Institute of Theoretical Astronomy (St.Petersburg) is maintained.

News on activity of our center is announced on our WWW page at http://ra.inasan.rssi.ru

\subsection{Miscellaneous}

In addition to the reports from the above-mentioned data centre, we can report the activity of two centres dedicated to HST data and specific telescopes, that of the Canadian Astronomical Data Center, reported by D. Crabtree and the ESO and ST-ECF Science Archive Facility, reported by B. Pirenne. The CADC continues to develop advanced archiving facilities using state-of-the-art technology. The CADC currently archives data from the Hubble Space Telescope and the Canada France Hawaii Telescope. In the near future data from the James Clerk Maxwell Telescope will also be archived by the CADC. In the past two years advances were made in several areas, as for example extensive use of CD-ROM technology for storing astronomical data, the implementation of On-the-Fly Calibration (OTFC) for data in the Hubble archive.

The ESO and ST-ECF Science Archive Facility (ESSAF) is a joint collaboration of the European Southern Observatory (ESO) and the Space Telescope - European Co-ordinating Facility (ST-ECF). Their mandate is the storage and re-distribution of data from ESO NTT and VLT telescopes as well as from the HST. The archives are composed of catalogues that can be browsed and of bulk data that can be requested. Among recent developments are the on-the-fly re-calibration of HST data.

Access to the facility is available from the World-Wide Web at the following address: http://archive.eso.org/ 


\section{REPORTS FROM WORKING GROUPS AND TASK GROUPS}

Before reporting on the activity of the Commission's Working Groups and Task Groups I would mention the activity of two Working Groups of two other commissions that receive our report. The first one, radioastronomical databases, is chaired by Dr. Andernach. He has developed a database which, in May 1996 contained 310 data sets with $\sim 1.7$ million entries, of which only a very minor fraction has been integrated in commonly used databases like NED, SIMBAD, LEDA and ESIS. Some 66 of these tables with over half a million entries are searchable through the "Einstein On-line Service" (EOLS) maintained at the Center for Astrophysics. Lack of funding has stopped further development of this service early in 1995. Since 1996 Dr. H. Andernach collaborates with Russian radio astronomers to build a prototype of the planned database, and partial data are now accessible via WWW from a server in St.-Petersburg. The second is the Working Group on Spectroscopic Data Archives, chaired by Dr. E. Griffin. Its chief activity was mainly directed to emphasise at various international meetings the important role of these archives.

The chairperson, Dr. E. Raimond, of the Working Group on Astronomical Data, has established a very complete activity report for the 20th CODATA General Assembly. This report can be obtained directly from Dr. Raimond or the commission's president. It is an excellent overview of the activity of astronomers in the field of Data and Information Handling.

\subsection{Working Group on Astronomical Data (E. Raimond)}

Maintenance of usable observatory archives is slowly becoming fairly common. In the past only very few observatories archived their data in such a way that other users than the original observers can use the data in scientific projects. Traditionally, proper archiving was more common with radio observatories than with optical ones. By now, the Space observatories (notably the International Ultraviolet Explorer (IUE) and the Hubble Space Telescope (HST)) and almost all modern ground-based observatories either have well designed archiving systems or are aiming towards that goal. A good example of a new observatory where archiving is properly built into the system is the Italian Galileo Telescope (TNG) (information by Fabio Pasian).

The TNG archiving activity and the development of the data reduction software are described in 5 documents produced by the Working Group on TNG archives. (http://www.pd.astro.it/TNGArchive/WG/WG.html). The "Cyclic Operation Scheme for the TNG" document describes the end-toend data flow concept for TNG. Additional information is available in:

http://www.oat.ts.astro.it/ pasian/aat_status.html. The page points to a more recent version of the design document, and to two other papers on the topic. There are furthermore a couple of other Web pages describing the TNG archiving simulation activity.

The OAT (Trieste) is developing the data reduction software for the Low Resolution Spectrograph for TNG; we plan to implement sub optimal pipeline processing aimed at a "quick-reduction" at the telescope, to evaluate the scientific quality of data while observing. Details can be found on a paper one can download from the Web page: http://www.oat.ts.astro.it/ pasian/newpapers.html

We can also mention the photometric database developed at the Institut d'Astronomie de l'Université de Lausanne which is accessible on-line through the Web (http://obswww.unige.ch/gcpd/)

\subsection{Working Group on Information Handling (A.G. Hearn)}

In the period up to 1 st July 1996, the working group has devoted its energy exclusively to the problems of electronic publication of joumals, and in particular to the organisation of a proposal for a one day Joint Discussion at the next General Assembly of the IAU at Kyoto in August 1997. The provisional programme can be found on URL http://astro.fys.ruu.nl:8000/iau/.

The working group was formed after the General Assembly in the Hague in August 1994 as a working group of Commission 5. It has now 26 members from 12 different countries. A rather intensive exchange of e-mails over a period of half a year was used to discuss the problems and prospects of electronic publication, and to formulate subjects that should be discussed during the Joint Discussion. A 
SOC for the Joint Discussion was then formed with 11 members from 8 different countries. The proposal for the Joint Discussion was then intensively discussed over a period of months.

The proposal for a Joint Discussion on electronic publishing has been accepted by the Executive Committee of the IAU, and is tentatively scheduled for Monday, 25th August 1997.

\subsection{Working Group on Libraries (W.H. Warren, B. Corbin)}

The JWGAL was created to foster closer co-operation between Commission 5 and the astronomical librarians around the world. A second colloquium on Library and Information Services in Astronomy (LISA II) was held at the European Southern Observatory in Garching on 10-12 May 1995 and discussions have begun about the possibility of a third meeting in 3 to 5 years. Ongoing activities among librarians have included the exchange of duplicates, discussions about the changing roles of libraries in the era of electronic journals, and how the libraries themselves fit into the overall picture of astronomical information services.

\subsection{Task Group on Designations (H. Dickel)}

The two main goals of the Task Group on Designations are 1) detecting ambiguous designations of sources of radiation outside the solar system and revising the "IAU Recommendations for Nomenclature" in order to help avoid such problems in the future and 2) promoting these "Recommendations".

A major accomplishment was the completion of the "Second Reference Dictionary of the Nomenclature of Celestial Objects" by Lortet, Borde, and Ochsenbein and its availability "on line" in SIMBAD.

The Task Group revised the "Specifications concerning designations for astronomical radiation sources outside the solar system" (e.g. "IAU Recommendations for Nomenclature"). Two different poster papers were presented, one at the ESO Workshop on "Handling and Archiving Data from Ground Based Telescopes" and another at Joint Discussion 20 on "The status of archiving astronomical data" at the IAU General Assembly. Further small changes were made to the "IAU Recommendations for Nomenclature" document and with the aid of F. Ochsenbein of CDS, it was placed on the World Wide Web (WWW URL address: http://cdsweb.u-strasbg.fr/how.html) along with a shorter document "How to refer to a source or designate a new one" (WWW URL address: http://cdsweb.u-strasbg.fr/iauspec.html).

"The Second Reference Dictionary ..." of acronyms is also now available on the WWW (URL address: http://astro.u-strasbg.fr/cgi-bin/Dic). The "How to ..." document has been published in a number of Observatory and Society Newsletters and a link to the "IAU Recommendations ...." appears on the home pages of several individuals, organisations, and observatories. A Poster on designations was presented at the LISA II Symposium in May 1995 and copies of the "How to ... " document have been passed out at several IAU symposia and other such meetings during 1996. Several Editors have published or agree to publish the "IAU Recommendations ..." or the "How to ... " document in their astronomical journals (such as A\&A, AJ, PASP). We are hopeful that others will at least include the URL addresses in their instructions to authors.

During the academic year 1995-96, the Task Group undertook a pilot program - a "Proposal for Registering a New Acronym". F. Ochsenbein, S. Borde, and P. Dubois were instrumental in setting up the necessary infrastructure and implementing it on the WWW as part of the "Second Reference Dictionary ...". Tucker of the Las Campanas Redshift Survey submitted the first acronym, LCRS, to this experimental registry. The "Clearing House" (an advisory subgroup of the Task Group) which screens the entries, will be developing further guidelines regarding acceptance of, and resolution of conflicting submissions, etc.

During the year preceding the 23rd IAU General Assembly in Kyoto and beyond, the Task Group will continue "advertising" the "IAU Recommendations for Nomenclature" in Newsletters and at Symposia and will alert Principal Investigators of on-going major astronomical surveys to the possibility of "Registering a New Acronym". A new initiative will be pursued in co-operation with journal editors 
and data centers conceming the feasibility, development, and implementation of "an automatic electronic check of non-conforming designations" relative to the "IAU Recommendations for Nomenclature" and the acronyms already in "The Second Reference Dictionary ..".

\subsection{Task Group on Data Centres and Networks}

Dr. F. Genova, Director of the CDS since July 1995, was coopted as a new member, and replaced Dr. M. Creze (who took a new position at the University of Vannes, France) as the chairperson of the TG, in March 1996.

Several members of the TG took a very active part in the organisation of the Colloquium "International co-operation in Dissemination of the astronomical Data", that was held in St Petersburg, Russia, on July 2-9, 1996, in particular Dr. O.B. Dluzhnevskaya, who was the co-chairperson of the Scientific Organising Committee with Dr. B. Hauck, and Drs. F. Genova, K. Nakajima and N.G. Roman as members of the Scientific Organising Committee. The Data Center activities, the diffusion of data, catalogues, the new possibilities offered by electronic publication, ... were thoroughly presented and discussed at the Colloquium.

A meeting of the TG was held in St Petersburg on July 5th. The Directors of the five Astronomy Data Centers (China, France, Japan, Russia, USA) and key personnel attended the meeting. A very fruitful discussion about the Data Center co-operation and common procedures was held, in particular about the standard description of Tables, common to the Data Centers and to the publishers for electronic publication. The TG will submit a resolution to the General assembly, assessing the role of the Data Centers for Astronomy.

\subsection{Task Group on UDC52 (G. Wilkins)}

The task of preparing proposals for the revision of the schedules for astronomy in the Universal Decimal Classification was accepted by Commission 5 at the Hague General Assembly. In the absence of active support from members of the Commission, the bulk of the work so far has been carried out at home by the chairman. One session at LISA II, organised by R M Shobbrook, was devoted to the revision of UDC 52. As a consequence, a group of "Friends of UDC 52", who are mainly astronomy librarians, was set up. The communication between the members about the proposals (to which the members have access by 'anonymous ftp') is mainly by e-mail. The members also have access to a draft guide on the use of UDC in astronomy, which was prepared, but not published, at the time of the last revision in 1974.

Reports on progress have been published by the UDCC in "Extensions and Corrections to the UDC" in 1994 and 1995. "The Astronomy Thesaurus", which was published in 1993, has been used to prepare a greatly expanded list of terms to be classified. The schedules for the classes 520/524 and for the special hyphen-auxiliaries have been revised in an attempt to cater for the new terms. Some new subdivisions have been proposed, especially for the hyphen auxiliaries. The aim is to produce a schedule that will be appropriate for two different purposes. Firstly, for the arrangement of books and other materials in libraries and other collections where browsing by the user is a valuable means of access to knowledge and information that would not otherwise be noticed. Secondly, to provide a tool for use in computerised systems for information retrieval that will supplement the simpler and more restricted lists of terms in the Astronomy Thesaurus and in other listings of keywords.

The British Standards Institution is willing to publish a special subject edition of UDC for astronomy; this will include the classification for astronomy and other relevant topics, an alphabetical index, a guide to the use of UDC, and various lists that will be useful for librarians and others who wish to use the classification. The chairman of the Task Group would welcome further assistance from astronomers and others who are willing to help in this project. It is desirable that UDC 52 and the Astronomy Thesaurus should be compatible and should be maintained regularly to take account of new terms and of our better understanding of the relationships between the objects and concepts that are represented in the classification. Consideration should be given to establishing for this purpose a Task Group that would include members of the IAU and other interested organisations.

B. Hauck President 